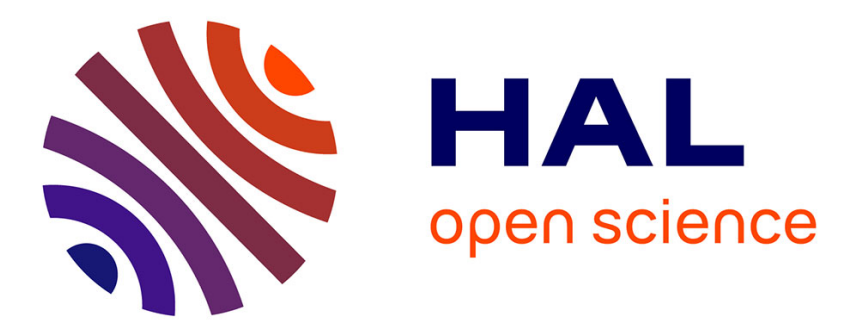

\title{
Optimizing the operation of an electrostatic precipitator by developing a multipoint electrode supplied by a hybrid generator
}

Antoine Silvestre de Ferron, Laurent Pecastaing, Thierry Reess, P Pignolet, F Lemont

\section{To cite this version:}

Antoine Silvestre de Ferron, Laurent Pecastaing, Thierry Reess, P Pignolet, F Lemont. Optimizing the operation of an electrostatic precipitator by developing a multipoint electrode supplied by a hybrid generator. Journal of Physics D: Applied Physics, 2009, 10.1088/0022-3727/42/10/105504 . hal03020514

\section{HAL Id: hal-03020514 \\ https://hal-univ-pau.archives-ouvertes.fr/hal-03020514}

Submitted on 23 Nov 2020

HAL is a multi-disciplinary open access archive for the deposit and dissemination of scientific research documents, whether they are published or not. The documents may come from teaching and research institutions in France or abroad, or from public or private research centers.
L'archive ouverte pluridisciplinaire HAL, est destinée au dépôt et à la diffusion de documents scientifiques de niveau recherche, publiés ou non, émanant des établissements d'enseignement et de recherche français ou étrangers, des laboratoires publics ou privés. 


\title{
Optimizing the operation of an electrostatic precipitator by developing a multipoint electrode supplied by a hybrid generator
}

\author{
A Silvestre de Ferron ${ }^{1}$, T Reess ${ }^{1}$, L Pécastaing ${ }^{1}$, P Pignolet $^{1}$ and F Lemont $^{2}$ \\ ${ }^{1}$ LGE, Université de Pau, 2 avenue Angot, 64000 Pau, France \\ ${ }^{2}$ CEA Marcoule, Centre de la Vallée du Rhône, 30200 Bagnols-sur-Cèze, France
}

\begin{abstract}
The authors investigated and improved the filtration efficiency of an electrostatic precipitator (ESP). A laboratory-scale pilot unit was developed to allow experimentation under conditions approaching those of the industrial ESPs used by the CEA at Marcoule (France). After elucidating the electrical phenomena and optically analysing the physical processes occurring inside the precipitator, a specific electrode was developed for use with a hybrid power supply. The experiments were based on analysing the variation over time of the electric charge injected into the particle separator, the particle mass collected at the ground electrode and the charges quantity measured on a grid in the airstream after the electrode unit. Photos were also taken under different electrical discharge conditions. The results show that combining a multipoint electrode and a hybrid generator $(30 \mathrm{kV}$ dc and $30 \mathrm{kV}, 1 \mathrm{kHz})$ improves the process efficiency and significantly extends the time frame (more than $10 \mathrm{~h}$ ) during which the process operates at optimum efficiency.
\end{abstract}

(Some figures in this article are in colour only in the electronic version)

\section{Introduction}

Electrostatic precipitators (ESPs) are frequently used in industry to eliminate solid particles from a gas stream. Such particulates are found mainly in the metallurgical industry, in road traffic, or in industrial waste incineration processes.

The physical principle of electrostatic precipitation has been extensively investigated [1,2], and is based on four mechanisms: charge creation in the gas, charge transfer to dust particles, drift of the charged particles in an electric field and collection of the charged particles on a grounded metal electrode.

Rising atmospheric pollution has led to stricter emission standards for gaseous effluents, and thus to higher performance requirements for ESPs. The two main requirements concern the filtration efficiency and problems of decreasing efficiency over time arising from back corona phenomena [3].

Several studies $[4,5]$ have evaluated the influence of the electrohydrodynamic (EHD) flows on particles transport and on ESP collection efficiency. It is generally believed that this flow structures reduce the efficiency of ESPs by increasing turbulent dispersion mechanisms. The aim of this work is to study the interaction between particles and discharge mode in an experimental way. The study of the temporal evolution of discharge characteristics on a single point-to-plane makes it possible to analyse the filtration process under specific hydrodynamic conditions.

The CEA's Marcoule centre has developed processes for solid waste treatment [6] by incineration in two steps (IRIS) or by incineration/vitrification in molten glass (SHIVA). These processes generate particle-laden off-gases that require electrostatic precipitation prior to stack release. The ESPs used for this purpose implement wire-cylinder geometry supplied by a dc voltage with negative polarity. The particle size and composition are very specific: they arise from the incineration of plastics (polystyrene, PVC, etc) or from the volatilization of some elements in the molten glass.

The purpose of this study was to improve the filtration performance of the ESP by simultaneously modifying the shape of the high-voltage electrode and the power supply 

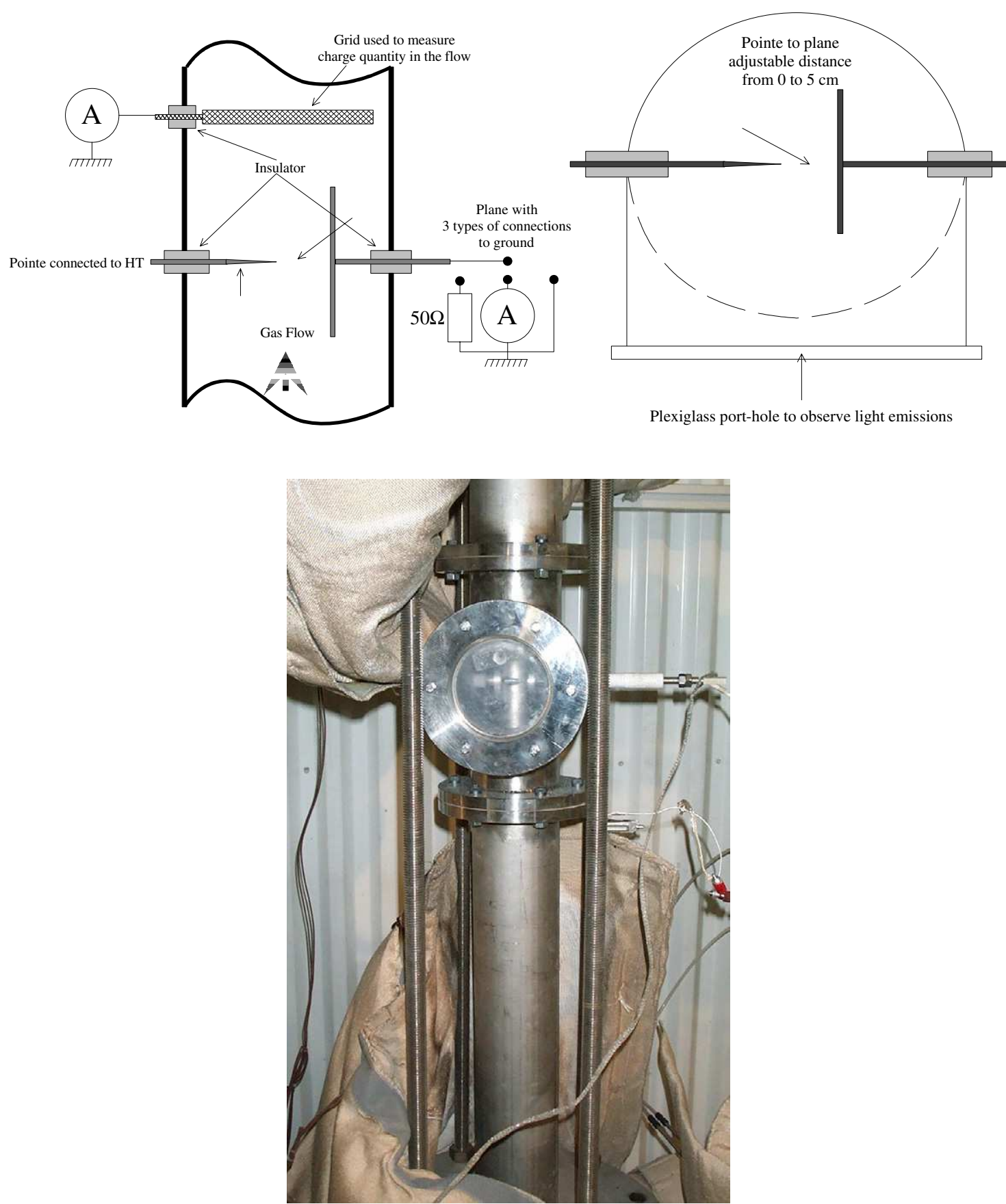

Figure 1. Principle schematic and photograph of the point-to-plane system.

characteristics. In the light of prior experimental results [7-10] we chose to work with a multipoint electrode with a hybrid power supply comprising high-voltage pulses superimposed on a dc voltage. This experimental study was carried out on a reduced-scale ESP installed at the Electrical Engineering Laboratory of the University of Pau, but of the same design as the one used at Marcoule.

The study was carried out in two stages. First, the variation over time of the physical quantities characterizing the filtration efficiency was determined using an ESP comprising a single point-plane device supplied with a negative dc voltage in order to observe the variation of the electrical parameters and analyse the electrical discharge phenomena during a complete filtration sequence. The second phase consisted of optimizing the multipoint electrode and evaluating the improved efficiency provided by the hybrid power supply.

\section{Experimental device}

The ESP is installed in an air loop with regulating systems for temperature $\left(130^{\circ} \mathrm{C}\right)$ and flow rate $\left(120 \mathrm{~m}^{3} \mathrm{~h}^{-1}\right)$. The ground electrode is a cylinder $1400 \mathrm{~mm}$ in height and 100 or $300 \mathrm{~mm}$ in diameter. The $100 \mathrm{~mm}$ diameter cylinder was modified to obtain the point-plane geometry shown in figure 1 used during the first part of the study. The plane can be grounded in three ways: via an ammeter to measure the mean current 


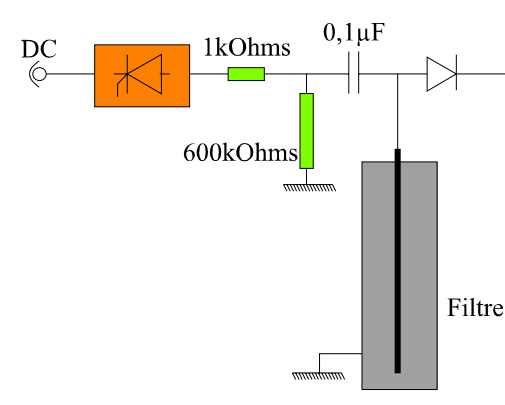

(a) Schematics

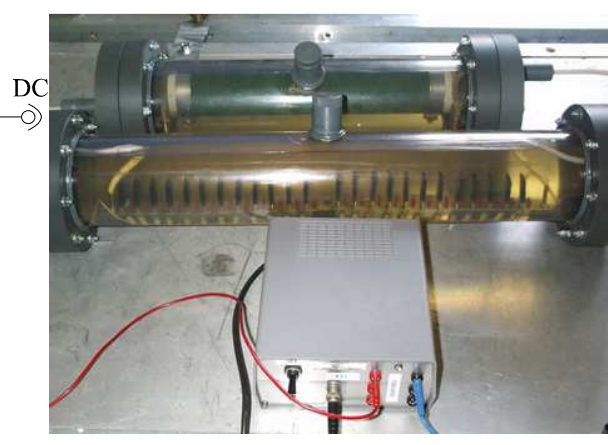

(b) photograph

Figure 2. Hybrid pulsed power/direct voltage supply description.

intensity, via a $50 \Omega$ shunt to measure the instantaneous current or by a direct connection. A grid connected to a picoammeter (electrometer) is placed downstream from the precipitator to measure the charges quantity in the airstream after the test unit. The cylinder includes a viewport to observe glow discharge phenomena.

The second study was carried out using a cylinder $300 \mathrm{~mm}$ in diameter with two HV electrodes: a conventional stainless steel wire $8 \mathrm{~mm}$ in diameter, and a multipoint electrode developed and optimized in our laboratory [11]. A preliminary study was carried out to measure the influence of the transverse and longitudinal point distributions on the mean current released in the precipitator. The results were used to design an electrode known as the 'heritrode', consisting of a copper tube $30 \mathrm{~mm}$ in diameter with points $30 \mathrm{~mm}$ long in a specific arrangement along the electrode.

The dust particles used for the tests were representative of those encountered in industrial applications at Marcoule. Dust particles were mixed with dry air and injected into the loop with a particle density of $2 \times 10^{6}$ particles $1^{-1}$ corresponding to a concentration of $0.06 \mathrm{~g} \mathrm{~m}^{-3}$. The filtration efficiency was determined using two particle counters (Grimm Aerosol Technik GmbH \& Co. KG) to measure the instantaneous particle concentrations at the precipitator inlet $\left(M_{\text {inlet }}\right)$ and outlet $\left(M_{\text {outlet }}\right)$. The two measurement points were situated in a laminar flow zone far from any sources of turbulence (heater, duct elbow, etc) and where the particle dispersion was assumed to be homogeneous over the flow cross section. The filtration efficiency $\eta$ (in percent) was determined from the following relation:

$$
\eta=\frac{M_{\text {inlet }}-M_{\text {outlet }}}{M_{\text {inlet }}} \times 100 .
$$

Two negative HV power supplies were used: a simple $50 \mathrm{kV}$, $40 \mathrm{~mA}$ dc supply and a hybrid pulsed dc supply. In the hybrid power supply unit, voltage pulses are generated by capacitors discharging into a resistor in parallel with the ESP (figures 2(a) and $(b)$ with switching by thyristors connected in series. The thyristors were triggered by fitting each of them with a ferrite core used such as a pulse transformer [12]. A dc voltage was applied to the HV electrode via diodes. This arrangement allowed the dc voltage to be modified simultaneously with the amplitude and frequency of the voltage pulses.

\section{Experimental results in point-plane geometry}

The purpose of the first study was to analyse the electrical behaviour of the electrostatic precipitation device with a simple point-plane electrode system in the airflow. The point radius was $200 \mu \mathrm{m}$ and the plane diameter was $90 \mathrm{~mm}$. The gap length $D$ ranged from 5 to $50 \mathrm{~mm}$. The system was operated on negative dc power to elucidate the back corona and reentrainment phenomena that can diminish the process efficiency. A conventional point-plane system could thus be used for mean or instantaneous current measurements, allowing the results to be compared with the published data $[13,14]$. The grid, connected to an electrometer, was positioned in the airflow downstream from the point-plane device to obtain image of the quantity of electrostatically charged particles that were not collected on the plane. Image analysis was performed using a digital camera containing a CCD sensor with the following characteristics: focal ratio: $1: 2.5$; CCD sensitivity: ISO 100; exposure time: $20 \mathrm{~s}$.

\subsection{Physical parameter variations over time}

First of all, an observation of the plane collection zone geometry leads to two conclusions: it can be noted the large size of the adhering particle spot $(8 \mathrm{~cm}$ in diameter) compared with the electrode gap $(1 \mathrm{~cm})$ and point diameter (few hundred micrometres). Moreover, its contours are remarkably sharp, revealing a major discontinuity in the density of the particle deposits.

3.1.1. Mean current. For the same experimental pointplane configuration $(D=10 \mathrm{~mm})$, figures $3(a)$ and $(b)$ show the variation over time of the mean current measured on the plane and on the grid, respectively. Two curves are plotted in each figure, representing the bounding limits on the rapid oscillations recorded by an $X-Y$ plotter. The variations over time can be analysed by dividing the curves into three zones separated by the two inflection points (at $t=8 \mathrm{~min}$ and $t=19 \mathrm{~min})$ :

- Zone 1. Both currents are of low intensity; the grid current corresponds to the zero-voltage value, indicating that all the electrically charged particles were trapped and precipitated mainly on the plane. 


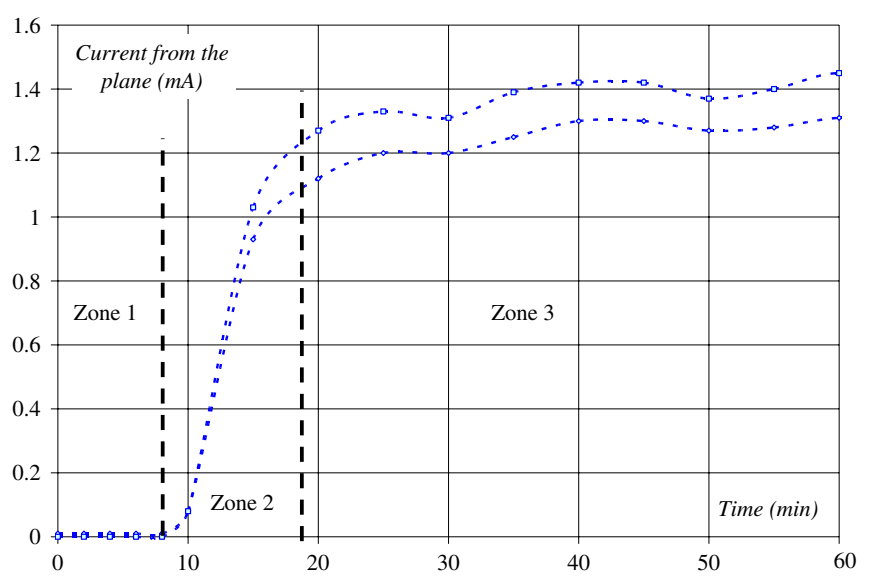

(a) Mean current measured on the plane

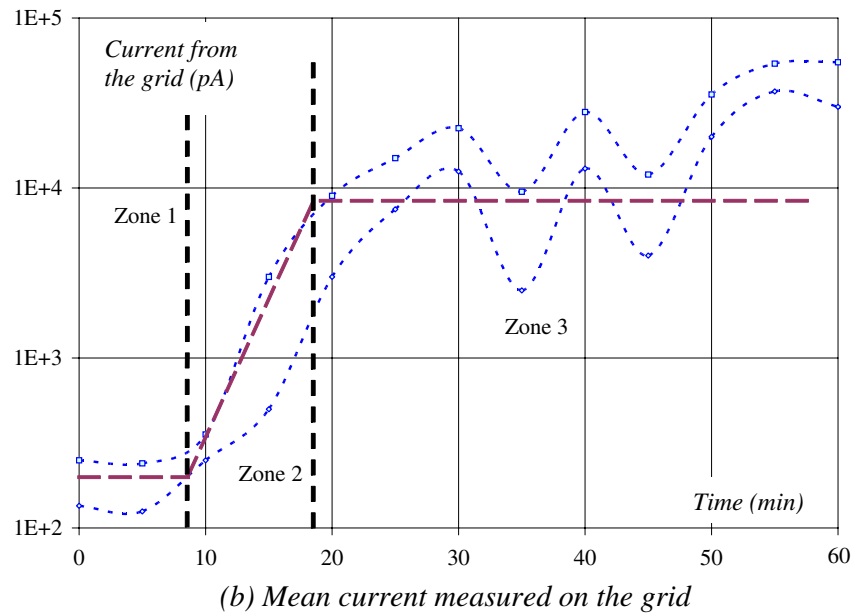

Figure 3. Temporal behaviour of mean currents in a point-to-plane geometry $(D=10 \mathrm{~mm})$

- Zone 2. The plane electrode current increases as a linear function of the precipitation time. At the same time, the grid current begins to increase due to the arrival on the grid of charged particles that were not collected on the plane electrode.

- Zone 3. The plane electrode current remains practically constant $(I=1.3 \mathrm{~mA})$; the grid current increases significantly to a maximum value of $10 \mu \mathrm{A}$.

3.1.2. Particle mass trapped on the plane. The objective was to analyse the particle mass trapped on the plane electrode versus the injected electric charge. A thin copper plate was mounted on the plane electrode, and the deposited particle mass was weighed after operating the unit for different periods of time. The corresponding electric charge was calculated by integrating the mean current. The results are shown in figure 4 for operating times ranging from 15 to $120 \mathrm{~min}$. Three zones clearly appear on this curve:

- The first (zone 1) corresponds to a linear mass increase versus the charge quantity. The slope of the curve is representative of the collection efficiency of the pointplane device. Although this value is practically constant, the actual collection efficiency $\eta$ cannot be measured using particle counters because of the small filtered air volume.

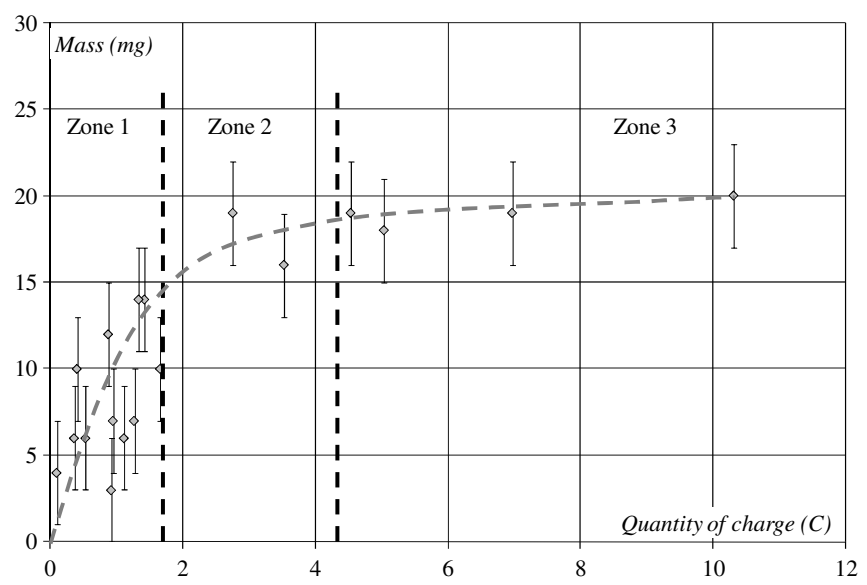

Figure 4. Mass of particles as a function of the charge quantity injected into the ESP.

- In the second zone the collected particle mass variation diminishes as the collection efficiency decreases. This is consistent with the strong increase in the grid current measured during this phase (figure $4(b)$-zone 2 ). It is also important to note that on the time scale the second zone coincides with the appearance of a luminous discharge recorded on the plane electrode, as discussed in section 3.3. This discharge is the beginning of a back corona phenomenon results in an increase in the plane electrode current (figure 4(a)—zone 2).

- The third zone is characterized by a flat region in the curve for particle mass versus charge; the collection efficiency is therefore near zero. The back corona effect reached a maximum at this point, and both recorded currents were constant.

Additional tests showed that the variations of these quantities over time were similar when the electrode gap was increased to distances near those found in the CEA Marcoule precipitator $(D=110 \mathrm{~mm})$.

\subsection{Optical parameter variations over time}

Figure 5 shows the discharge observed in the same experimental device. The photos also reveal three phases corresponding to the zones described above:

Phase 1. Figure 5(a), corresponding to phase 1, shows the characteristic Trichel discharge at the point. A blue glow appears on the plane after $30 \mathrm{~s}$, then phase 1 ends with a diffuse filamentary discharge corresponding to a current peak and an intense anode spot appears on the plane opposite the point (figure 5(c)).

Phase 2. In phase 2, a discharge occupies a conical volume extended from the point to the plane. The discharge colour is more reddish than the glow. The luminous intensity of the cone increases as diffuse channels develop between the two electrodes inside the luminous cone. This phase ends with the establishment of several tens of channels with a very luminous spot at each end (figure 5(e)). The semi-spherical 


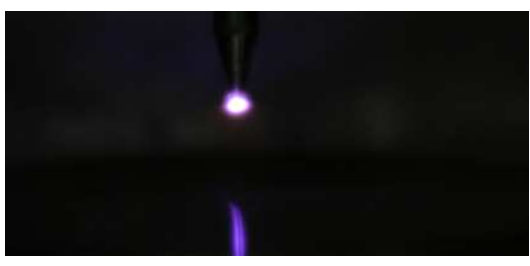

(a) $t=0$ min and $I=0.06 \mathrm{~mA}$.

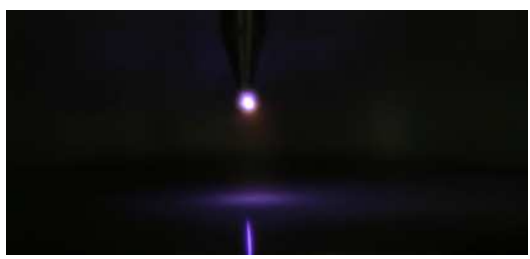

(b) $t=15 \min$ and $I=0.08 \mathrm{~mA}$

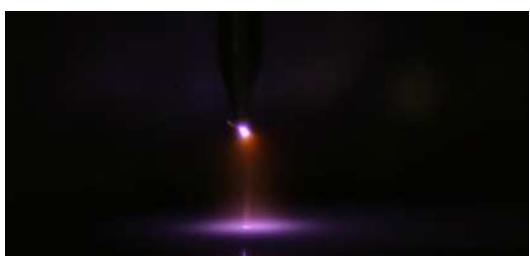

(c) $t=30 \mathrm{~min}$ and $I=0.15 \mathrm{~mA}$.

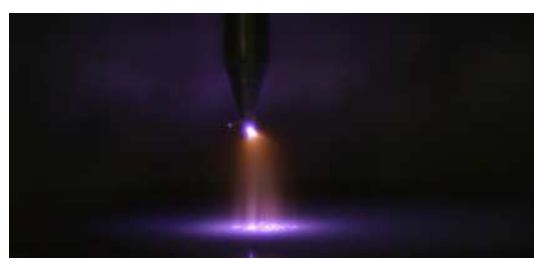

(d) $t=60 \mathrm{~min}$ and $I=0.37 \mathrm{~mA}$.

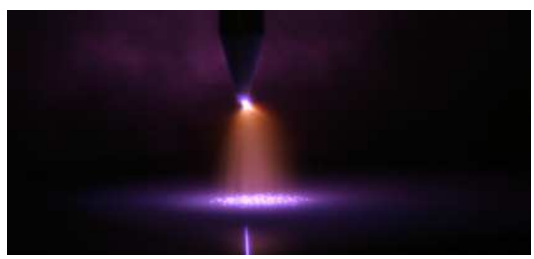

(e) $t=90 \mathrm{~min}$ and $\mathrm{I}=0.58 \mathrm{~mA}$

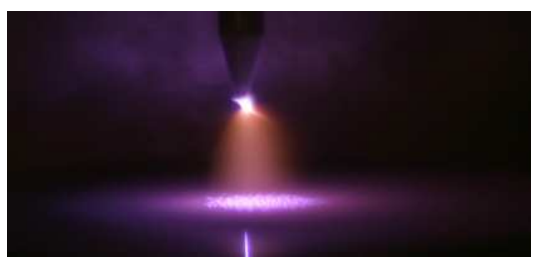

(f) $t=120 \mathrm{~min}$ and $I=0.85 \mathrm{~mA}$

Figure 5. Photographs of optical phenomenon occurring between a single point and a plane during filtration activity.

plume characteristic of Trichel pulses also disappears during this phase. The cathode glow is very diminished, indicating the end of conventional Trichel conditions.

Phase 3. Phase 3 corresponds to an intense luminous emission (figure $5(f)$ ) including:

- a blue glow on the plane electrode (anode) with a radius largely exceeding the electrode gap $(10 \mathrm{~mm})$,

- a luminous cone extending from point to plane,

- diffuse channels within the cone, ending in high-intensity spots of light,

- a significant increase in the brightness of the cathode spot compared with the preceding phase.

\subsection{Interpretation of results}

The analysis and interpretation are subdivided into three parts corresponding to the three phases and related to the three zones described above. Figure 6 summarizes these observations.

3.3.1. Phase 1. During phase 1, the mean point-to-plane current increased slightly and the grid current remained very low (a few picoamperes). The discharge conditions were characterized by a luminous ball at the point, followed after a few seconds by luminous discharges on the plane. The instantaneous current recording showed that the point discharge conditions were initially a Trichel pulse regime that quickly (within a few seconds) became continuous. The blue halo could be a micro-discharge zone occurring as soon as a thin layer of particles was deposited [15]. Because of the space charge zone above the deposit, these micro-discharges very sharply delimited the particle collection zone near the plane electrode $[16,17]$. The brightness of the micro-discharges varied over time, suggesting that the conditions were modified as the particle deposit became thicker leading to phase 2 . It may be noted that these phenomena had no effect on the point discharge conditions. This implies that under steady-state particle injection conditions phase 1 corresponds to a linear mass variation, and therefore to constant, optimum efficiency (figure 6-zone 1).

3.3.2. Phase 2. During phase 2, we also observed the mean point-to-plane current and the grid current. The point-to-plane current increased significantly after $20 \mathrm{~min}$ of operation of the pilot ESP. At the same moment, the grid current began to increase slightly.

On the photos we observed the appearance of the first diffuse filamentary discharge characterized by a very bright spot on the deposit, with an orange column above it. These discharges from the plane electrode became increasingly numerous, and the point conditions changed from a Trichel ball to a smaller but higher intensity zone indicating the end of the classical Trichel regime. These observations suggest that the filamentary discharges were due to penetration of the insulating layer formed by the particle deposit on the plane electrode. The thickness of the particle layer continually increased from the beginning of ESP operation, as did the surface deposit of negative charges, until the potential difference across the layer reached the point of dielectric breakdown. Diffuse filamentary discharges propagating into the gap led to a charge transfer that resulted in a higher mean current and could have modified the point discharge conditions. 

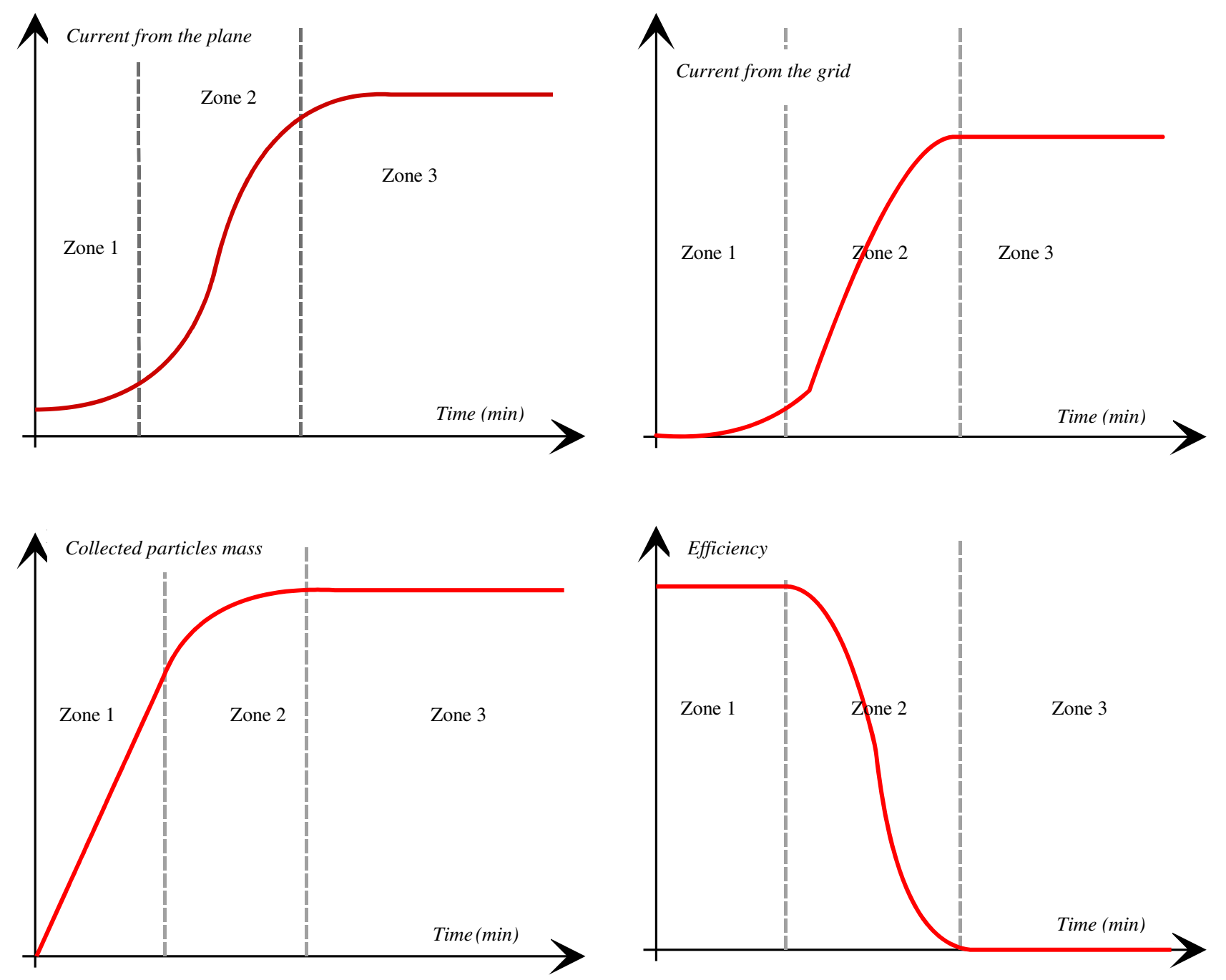

Figure 6. Schematic summary of observations.

The 'channels' that appear each time the deposit is perforated seem to be streamers between the two electrodes, but which are unable to induce short-range breakdown because the current is limited by the resistance due to the particle deposit on the point. This deposit can be attributed to the particle charge due to positive ions in the gap (arising from the plane electrode discharges and the point discharge regime) and their subsequent migration towards the cathode. The luminous column, which increased in volume over time, would therefore be plasma characterized by high-density charged species and a very weak field.

The multiplication of these phenomena would result in charged particles that do not drift towards the electrodes. This could account for the increasing grid current. The filtration efficiency of this system would therefore diminish during this phase, and the mass variation would therefore no longer be linear (figure 6-zone 2).

3.3.3. Phase 3. The mean current in the gap becomes constant during phase 3, and the grid current increases significantly. The instantaneous current bounding measurements reveal oscillations corresponding to current peaks. Optically it appears that the large number of channels formed during the preceding phase is consolidated into a single orange column. At the beginning of this phase the collected particle mass opposite each point also appears to reach a saturation value that remains constant.

The column can be explained in the same way as for the channels described above, by the existence of plasma between the two strong fields near the two electrodes. Dielectric breakdown occurs on the plane electrode, the particle deposit on the point continues to limit the current. The field in the gap would then be insufficient for continued particle collection. The collection efficiency drops to zero, and the charged particles are attracted to the grid. This phase can continue as long as dielectric breakdown does not occur on the point electrode. As soon as the particle layer is breached, however, the current is no longer limited and arcing occurs. The breakdown occurs without any increase in voltage because of the very high gap ionization during all three phases.

It is therefore obvious that the current increase observed during phase 2 is undesirable for satisfactory operation of the ESP because all the positive discharges responsible for this increase lead to diminished collection and/or release of particles into the airstream with a resulting drop in 


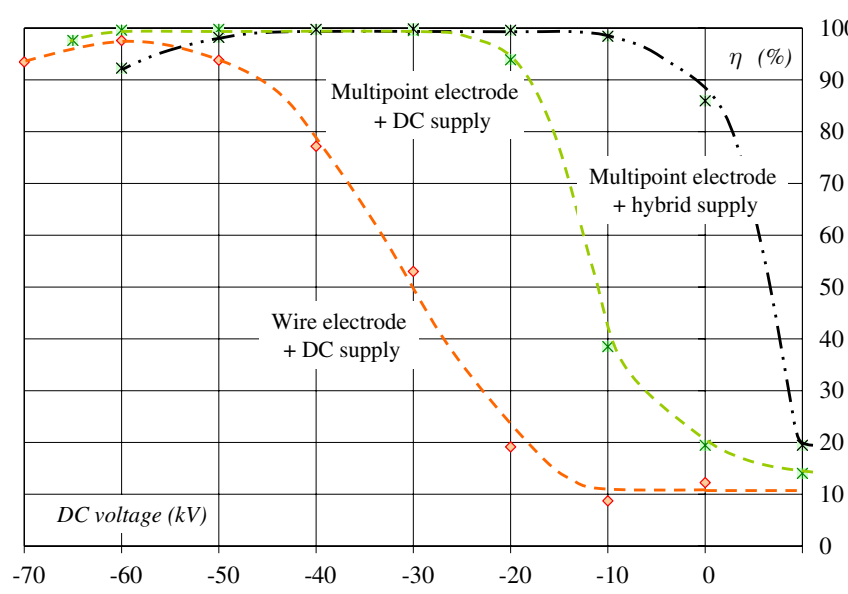

Figure 7. Precipitation efficiency as a function of applied voltage for the two electrodes of the ESP.

efficiency. Enhancing the filtration performance therefore implies increasing the current while remaining in zone 1 and avoiding the transition to zone 2 .

\section{Experimental results in the reduced-scale ESP}

We have shown that increasing the current intensity over time (zones 2 and 3 ) does not increase the precipitation efficiency. In addition, the precipitation efficiency is characterized not only by the slope of the curve for the particle mass trapped on the plane electrode versus the quantity of electrical charges (figure 6-zone 1) but also by the current intensity measured on the plane (figure $5(a)$-zone 1). Optimizing this current should result in optimization of the precipitation efficiency. We therefore measured the mean current supplied to the precipitator versus the shape and number of point emission sites on the HV electrode [10] and developed a new electrode with a large number of points distributed in an optimized pattern over the full length of the electrode: the 'heritrode'. The next step was to substantiate that the use of this type of electrode with a hybrid power supply could improve the precipitation performance. We first compared the instantaneous behaviour for only a few minutes to calculate the process efficiency. We then compared the process efficiency over longer time periods of up to several hours.

\subsection{Instantaneous precipitation efficiency}

Figure 7 shows the precipitation efficiency versus the applied voltage for three different configurations: the conventional wire-cylinder geometry used by the CEA, supplied by a negative dc voltage, and two configurations using our optimized 'heritrode' with dc voltage or hybrid voltage supplies. The hybrid voltage supply includes a variable dc component ( $X$-axis) superimposed on $1 \mathrm{kHz}$ pulses with an amplitude of $-30 \mathrm{kV}$.

Two issues must be considered. The first concerns loop operation without the application of any electric field: the precipitator efficiency under these conditions is between $10 \%$ and $20 \%$, because of the natural particle charge due to friction in the airflow. The second concerns the distinction between

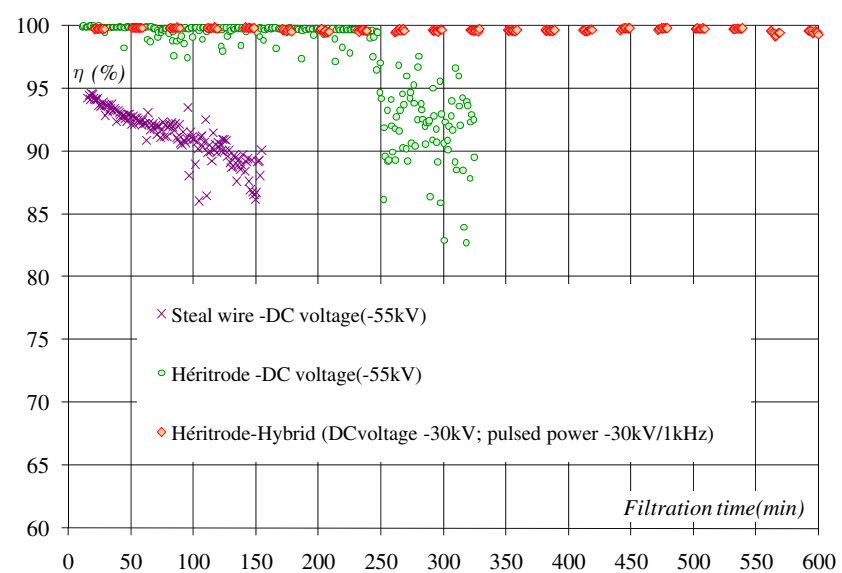

Figure 8. Temporal behaviour of the precipitation efficiency of the ESP.

the efficiency obtained with no dc or pulsed voltage applied, and the efficiency obtained without any dc voltage but with the pulsed power supply active; in this case the central electrode is supplied with a purely pulsed voltage $(-30 \mathrm{kV}, 1 \mathrm{kHz})$.

Figure 7 clearly shows that the combination of a multipoint electrode and hybrid power supply significantly improves the process efficiency. The optimum efficiency operating range is also broader. In addition, the diminished efficiency observed at high voltages is related to electrical arcing in the gap; this is a classic phenomenon with ESPs. A lower hybrid voltage would ensure optimum operation without arcing. The final step was to verify the device operation over time.

\subsection{Precipitation efficiency over time}

The same flow conditions and electrode/power supply combinations were used as for the previous tests. The hybrid signal was superimposed on a constant $-30 \mathrm{kV}$ supply.

The process efficiency is plotted versus the loop operating time in figure 8. It is interesting to note that throughout the tests no drop in efficiency was observed for the optimized electrode supplied with a hybrid voltage input, in contrast to the other two configurations. The process efficiency during these experiments ranged from $97.9 \%$ to $99.5 \%$, indicating that this electrode supplied by a hybrid generator maintains the device in zone 1 (figures 3(a), (b) and 4). As we assumed after investigating the point-plane device, the undesirable phenomena (back corona and particle detachment) never occurred and the ESP operated with optimum precipitation efficiency.

A final comparison about the multipoint electrode fouling after 320 min of dc operation and after more than $600 \mathrm{~min}$ with a hybrid supply shows that at the end of the dc experiment the electrode was fouled over $500 \mathrm{~mm}$, compared with only $40 \mathrm{~mm}$ at the end of the hybrid voltage operation. Fouling can be equated with the development of back corona and particle release on the collection electrode. We can conclude that a hybrid power supply minimizes the undesirable phenomena, resulting in higher process efficiency for extended operation. 


\section{Conclusion}

In order to investigate and improve the efficiency of ESPs in the CEA waste incineration off-gas stream we used a laboratoryscale pilot unit to conduct experiments under conditions approximating reality but using simple hydraulic, geometric and electrical parameters. The objective was to understand the electrical phenomena occurring inside an ESP and to propose an industrial device capable of operating at maximum efficiency (>99\%) for as long as possible.

To account for the operation of a multipoint electrode we simplified the problem using a reduced-scale model consisting of a point and plane with a narrow gap placed in the particle flow stream. Using this mockup cathode site, we measured the collected particle mass and the mean current, and obtained photos of the discharges over time. The system efficiency was explained by a description of the discharge conditions postulated in the gap. The buildup of a particle deposit on the plane electrode immediately forms a glow that appears to be attributable to micro-discharges related to the increased local electric field at the triple points, and which augments particle precipitation on well-defined zones. A second type of discharge subsequently occurs between point and plane, with a radical change in the Trichel regime that gives way to a positive column in the gap. Filaments between an anodic spots at the point of dielectric failures and the cathode zone are clearly visible in the images. The particles would thus be charged in this zone but would not migrate because the field is too weak. If these conditions are maintained the establishment of the column leads to a dielectric breakdown across the gap.

We designed a multipoint electrode, the 'heritrode', on which the point size, number and geometric distribution have been optimized. We have tested a device with a hybrid voltage, with $-30 \mathrm{kV}, 1 \mathrm{kHz}$ pulses superimposed on a $-30 \mathrm{kV}$ dc signal. With the multipoint electrode this operating mode extends the working voltage range to nearly $100 \%$ and should allow operation for $10 \mathrm{~h}$ or more without any drop in efficiency.

\section{References}

[1] Theodore L and Buonicore A J 1976 Industrial Air Pollution Control Equipment for Particulates (Boca Raton, FL: CRC Press) p 385
[2] Agamemnon A et al 2002 Prediction of the cleaning efficiency of an electrostatic precipitator $J$. Electrostatics 55 111-33

[3] Leonard G, Mitchner M and Self S A 1980 Particle transport in electrostatic precipitator Atmos. Environ. 40 425-31

[4] Soldati A 2000 On the effect of ElectroHydroDynamic flows and turbulence on aerosol transport and collection in wire-plate electrostatic precipitator J. Aerosol Sci. 31 293-305

[5] Yamamoto T, Morita Y, Fujishima H and Okubo M 2006 Three dimensional EHD simulation for point corona electrostatic precipitator based on laminar and turbulent models J. Electrostatics 64 628-33

[6] Lemort F, Charvillat J P and Longuet T 1999 Adapting Electrostatic Precipitator to the IRIS Nuclear Waste Incineration Process (Valhro/Marcoule, Commissariat à l'Énergie Atomique)

[7] Dinelli G et al 1990 Industrial demonstration of impulse energization on electrostatic precipitator Proc. $3 \mathrm{rd}$ Int. Conf. on Electrostatic Precipitation (Padua, Italia)

[8] Dinelli G et al 1990 Pulse power electrostatic technologies for the control of flue gas emissions J. Electrostatics $2523-40$

[9] Elayyan H S B, Bouziane A and Waters R T 2002 Theoretical and experimental investigation of a pulsed ESP J. Electrostatics 56 219-34

[10] Silvestre de Ferron A, Reess T, Pecastaing L, Gibert A, Domens P and Pignolet P 2008 Removal of diesel soot particles using an ESP supplied by a hybrid voltage Eur. Phys. J. Appl. Phys. 43 103-9

[11] Silvestre de Ferron A 2006 Utilisation des fortes puissances électriques pulsées dans l'électrofiltration d'effluents gazeux PhD Thesis University of Pau, France

[12] Bac J, Reess T, Pecastaing L, Paillol J and Domens P 2007 The use of commercial thyristors in repetitive high voltage switching devices for plasma sources Euro. Phys. J. Appl. Phys. 37 129-41

[13] Morrow R 1985 Theory of negative corona in oxygen Phys. Rev. A 32 1799-809

[14] Reess T and Paillol J 1997 The role of the electric field in Trichel pulse development in air at atmospheric pressure J. Phys. D.: Appl. Phys. 30 3115-22

[15] Prashanth S Kothnur and Laxminarayan L Raja 2005 Two-dimensional simulation of a direct-current micro-hollow cathode discharge J. Appl. Phys. 97 1-12

[16] Penache C et al 2000 Experimental Study of High Pressure Glow Discharges based on MSE Arrays (Hakone)

[17] Penache M C 2002 Study of high-pressure glow discharges generated by micro-structured electrode (MSE) arrays PhD Thesis Frankfurt University 